
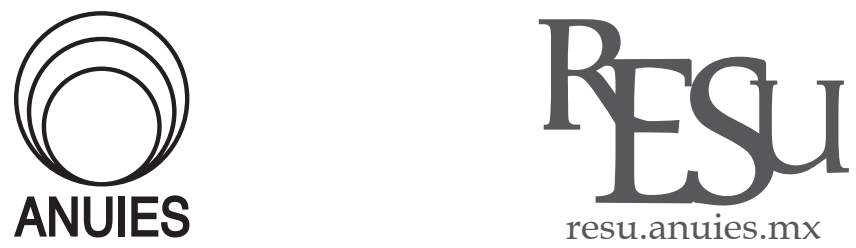

ARTíCULO

\title{
La formación de ingenieros en los modelos educativos del Tecnológico Nacional de México: Un significante ausente
}

\author{
Engineers Training in the Tecnológico Nacional de México Educational \\ Models: An absent signifier
}

\section{Magda Concepción Morales Barrera}

Centro Interdisciplinario de Investigación y Docencia en Educación Técnica

Correo electrónico: mmorales@ciidet.edu.mx

Recibido el 22 de marzo del 2018; aceptado el 03 de octubre del 2019

\section{Resumen}

Este trabajo plantea una lectura analítica de los modelos educativos que han orientado las concepciones y prácticas formativas en el ahora Tecnológico Nacional de México; a partir de ella se propuso dar cuenta, desde un abordaje hermenéutico, de las nociones de formación de ingenieros y de educación superior tecnológica presentes en dichos modelos. El resultado del análisis permite sostener la ausencia de esas nociones y por tanto de su problematización en los documentos revisados; en las conclusiones se bosquejan elementos que permiten comprender las implicaciones de esta ausencia para el Subsistema de Educación Superior Tecnológica en México.

Palabras clave: Formación de Ingenieros; Educación superior tecnológica; Modelo educativo; Organización curricular 


\begin{abstract}
This paper propose an analytical review of the educational models that guide the conceptions and formative practices in the Tecnológico Nacional de México; its desirable to understand notions about engineers formation and higher technological education that are present in the mentioned models from an hermeneutic approach. The analytical results indicate that these notions are absent and therefore such problematization. To conclude, it is proposed elements for the understanding of these absence's implications to this educational subsystem.
\end{abstract}

Keywords: Engineers formation; Higher technological education; Educational model; Curricular organization

\title{
Introducción
}

L a lectura analítica de los modelos educativos del Tecnológico Nacional de México (тесNм), que se propone en este texto, está enmarcada en el contexto de la investigación "La formación de ingenieros en el TecNM: Significaciones y Preocupaciones". La revisión de los modelos educativos en cuestión se realizó en un principio con la intención de rastrear las concepciones de formación de ingenieros presentes en los documentos que orientan las prácticas formativas del тесNM, intención guiada por la pregunta central implicada en el proyecto de investigación, a saber, ¿cuáles son las significaciones de ser ingeniero en el тесNм y qué implicaciones tienen en las prácticas educativas de sus actores?

La búsqueda de respuestas a esta pregunta permitió dar cuenta de que a pesar de ser el TесNM el subsistema de educación superior tecnológica más grande de México y más del 85\% de su oferta educativa de nivel licenciatura corresponde a ingenierías, formando en sus aulas al $44 \%$ de los ingenieros del país (TecNM, s/f), no hay trabajos de investigación o literatura académica enfocada en la cuestión de la formación de ingenieros en este subsistema o que discuta sobre la noción de educación superior tecnológica que lo direcciona; de ahí que surgiera la necesidad de revisar los modelos educativos ${ }^{1}$ que han enmarcado, en distintos momentos, el accionar de este subsistema educativo, con la finalidad de identificar los enfoques y tendencias de la for-

\footnotetext{
1 Modelo Educativo para el Siglo xxI; Modelo Educativo para el Siglo xxI, Formación y Desarrollo de competencias profesionales y Modelo de Educación Dual para nivel licenciatura del Tecnológico Nacional de México, aunque este último no es de observancia general, se tomó en cuenta por ser presentado como un nuevo modelo educativo.
} 
mación de ingenieros que estuvieran dando pauta a sus prácticas y procesos educativos.

Conforme se avanzó en el análisis de los Modelos mencionados, se realizaron hallazgos que si bien no responden directamente a la pregunta de investigación planteada ni permiten entender las concepciones sobre la formación de ingenieros o la educación superior tecnológica, significantes ausentes en los modelos revisados, sí procuran comprender esta ausencia y aportan al entendimiento de sus implicaciones.

Estos hallazgos son los que se consignan en este documento, a partir de los cuales se busca poner de relieve la necesidad de incorporar un posicionamiento conceptual sobre la formación de ingenieros en la base del modelo educativo para el TecNM, sin dejar de reconocer la importancia de los modelos educativos analizados que han configurado los primeros pasos hacia la organización académico-curricular del Subsistema.

Este trabajo analítico se realizó desde un enclave epistemológico basado en supuestos hermenéuticos, en tanto no partió de la pretensión de descubrir una propiedad inherente al objeto para enunciar una verdad absoluta e incuestionable, más bien estuvo direccionado hacia la construcción de interpretaciones que posibilitan un acercamiento a los significados de los textos, reconociendo el contexto y las condiciones de su producción; de esto resulta una lectura, entre muchas posibles.

El proceso analítico desarrollado es cualitativo y no lineal, parte de una mirada aporética que implica situarse más allá de las contradicciones y oposiciones que caracterizan las lógicas de pensamiento disyuntivas y dicotómicas, para entender distintas relaciones caracterizadas por tensiones en las que los opuestos se imbrican y constituyen mutuamente. Esto deviene en una lectura que se aleja de pretensiones clasificatorias y deterministas; más que señalar lo bueno o lo malo, o determinar lo correcto o incorrecto de los modelos educativos analizados, se enfatiza en las continuidades, rupturas y ausencias que configuran discursivamente los modelos que, como todo modelo, tienen fallas que pueden conformar espacios de posibilidad.

La estructura del artículo se compone de cinco apartados. El argumento central del primer apartado tiene que ver con la construcción histórica del тесNM; el segundo bosqueja las condiciones que generaron la necesidad de un modelo educativo que articulara las concepciones y prácticas educativas en el subsistema; el tercer articula los elementos que han estado presentes impregnando huellas en las estructuras y concepciones de los tres modelos existentes; el cuarto está dedicado a identificar tensiones, rupturas y contradicciones conceptuales; finalmente, en las conclusiones se plantean las implicaciones de la ausencia de problematización en torno a la formación de 
ingenieros y la educación superior tecnológica en los modelos educativos revisados y se proponen algunas líneas de acción hacía la construcción de una base conceptual que sostenga un modelo educativo del TесNM pertinente y relevante a las necesidades y exigencias actuales.

\section{Apuntes para pensar la construcción histórica del Tecnológico Nacional de México}

No se pueden analizar los modelos que recientemente han orientado las concepciones y prácticas educativas en los Institutos Tecnológicos (IT), hoy organizados y direccionados bajo la figura del $\mathrm{TeCNM}^{2}$, sin entender el contexto de su producción y su operación; por ello, en este apartado se exploran las condiciones históricas que enmarcan este contexto.

Constituido en 2014, el TecnM es actualmente la Institución de Educación Superior tecnológica más grande del país, con 266 campos (126 institutos tecnológicos federales, 134 descentralizados y 6 Centros) donde se atiende a una matrícula de 521,105 estudiantes de licenciatura y posgrado (тесNм, s/f). Detrás de estos datos y la afirmación de ser la institución más grande que ofrece educación superior tecnológica en México, hay una trayectoria histórica que es necesario reconocer para dimensionar las implicaciones políticas, económicas, culturales, sociales y educativas de este Sistema.

El trazado de esta trayectoria no puede ser lineal ni ascendente, está atravesada por tensiones, rupturas y giros que generan la posibilidad constante de reconstrucción, en tanto nada está escrito ni puede ser pre-visto. Estos apuntes intentarán dar pistas de ello y de sus profundas imbricaciones en la construcción histórica del país.

\section{Los primeros momentos (institucionalizados) de la educación superior tecnológica en México}

La historia de la educación superior tecnológica en México está anudada a la fuerte tradición de formación de ingenieros, inaugurada formalmente con el Real Seminario de Minería, convertido en 1792 en Colegio de Minería (Weiss y Bernal, 2013). Es relevante señalar que esta Institución tenía entre

\footnotetext{
2 Organismo desconcentrado de la Secretaría de Educación Pública, que sustituyó a la Dirección General de Educación Superior Tecnológica (DGEST).
} 
sus objetivos "difundir el conocimiento científico" e "instruir a la juventud novohispana en las técnicas más modernas de explotación y refinación de minerales" (Flores, 2004: 226), en un programa organizado en siete años, cuatro dirigidos a la formación teórica (Ciencias y artes mecánicas) y tres a la puesta en práctica de los conocimientos adquiridos (en los reales de minas novohispanos); al final de los siete años, los estudiantes tenían que elaborar una tesis para poder obtener el título de ingeniero.

En el seno del Real Seminario se debatían dos concepciones inconmensurables de la educación: una utilitaria, sostenida por algunos funcionarios y comerciantes que reducían la función de la Institución a la de un negocio, y cuestionaban el prolongado tiempo de formación y los cuantiosos recursos económicos destinados a ello, por lo que exigían que se generara utilidades a corto plazo; y una concepción apegada a la ciencia, sostenida por un grupo de profesionales mineros - influidos por la escuela europea - para quienes la ciencia era la pieza clave para resolver los problemas técnicos, de ahí que argumentaran la necesidad de respetar e incluso prolongar los tiempos de la formación teórica. Fausto de Elhuyar, el primer director del Real Seminario, posicionado en esta concepción, propuso extender los estudios teóricos a cinco años y reducir a dos los de práctica (Flores, 2004), a manera del modelo politécnico francés ${ }^{3}$; basado en este modelo, 1867 el Real Seminario de Minas se transformó en la Escuela Nacional de Ingeniería (Bazant, citada en Weiss y Bernal, 2013).

En 1822 se funda el Colegio Militar, dedicado a la formación de ingenieros especialistas en mecánica bélica, hidráulica y construcción (Weiss y Bernal, 2013), también conocido como Academia de Ingeniería cuyo propósito era impartir conocimientos científicos necesarios a los oficiales del ejército y a jóvenes interesados en la ingeniería militar. El Colegio Militar fue cerrado y abierto en diversas ocasiones, relacionadas con movimientos sociales $\mathrm{u}$ ocupaciones extranjeras (SEDENA, s/f), y fue hasta 1960 cuando la Institución adquirió cierta estabilidad al decretarse la fundación de la Escuela Militar de Ingenieros.

Otras escuelas de ingeniería que hay que mencionar entre los primeros momentos en la historia de la educación superior tecnológica en México, son la Escuela Práctica de Metalurgia y Labores de Minas, creada en 1877 en Pachuca, y la Escuela Especial de Ingenieros, concebida en 1883 (Weiss y Bernal, 2013).

Aunque cada una de las Instituciones que se han mencionado tuvo condiciones específicas y una historia de creación muy particular, por lo cual no

\footnotetext{
${ }^{3}$ Basado en la consideración de la supremacía del conocimiento científico (principalmente de las matemáticas y la física) sobre otros saberes, y el entendimiento de la técnica como resultante de la aplicación de este conocimiento.
} 
es posible generalizarlas, es importante enfatizar que en todas, de maneras más o menos visibles, hay huellas del pensamiento moderno y los principios ilustrados, los cuales construyeron la base de los anhelos de independencia y orientaron los primeros pasos de la reciente nación, explicitado en la constitución de 1824 que en su artículo 50 expresa:

Son facultades exclusivas del Congreso general:

1) Promover la ilustración, asegurando, por tiempo limitado, derechos exclusivos a los autores por sus respectivas obras, estableciendo colegios de marina, artillería e ingenieros, exigiendo uno o más establecimientos en que se enseñen las ciencias naturales y exactas, políticas y morales, nobles artes y lenguas, sin perjudicar la facultad que tienen las legislaturas para el arreglo de la educación pública en sus respectivos Estados.

Desde entonces, la educación superior tecnológica en México ha estado profundamente ligada a la historia de la nación, impregnada de sus anhelos y reflejando, como espejo, sus problemáticas; orientada, desde esos primeros momentos, por un ideal fundante, a saber, el constituirse como la base del desarrollo económico y social del país, partiendo de la adquisición y la aplicación del conocimiento científico.

\section{El Instituto Politécnico Nacional}

En 1936 se funda el Instituto Politécnico Nacional (IPN), que articuló diversas escuelas, formaciones y técnicas (Weiss y Bernal, 2013); el proyecto del IPN se pensó en 1932, con la idea de integrar y estructurar la enseñanza técnica. Se cristalizó gracias al apoyo de Juan de Dios Bátiz (Senador) y Lázaro Cárdenas del Río (Presidente de la República), cuyo planteamiento políticoideológico central consistía en desarrollar los postulados revolucionarios en materia educativa. Así se concibió así al IPN como motor de desarrollo y espacio de igualdad, asignándole dos tareas fundamentales: apoyar el proceso de industrialización del país y brindar alternativas educativas a los distintos sectores sociales, especialmente a los más desfavorecidos (IPN, s/f).

Es importante evidenciar dos tensiones constitutivas en este momento de la historia del IPN. La primera tuvo que ver con los ideales pos-revolucionarios y la tradición de la educación tecnológica basada en el modelo politécnico francés, presente desde los momentos fundacionales de la educación superior tecnológica en México y consolidada en el porfiriato, con la cual el IPN no estableció rupturas importantes (Weiss y Bernal, 2013), al menos en términos académico-pedagógicos. La otra se ubicó entre la tarea de brindar 
alternativas educativas a los sectores sociales más desfavorecidos y la centralización de su oferta educativa, en tanto que “[...] con excepción de algunas prevocacionales todas las escuelas con las que comenzó a funcionar el IPN estaban en la capital del país" ${ }^{4}$ (Weiss y Bernal, 2013: 154). Al respecto, conviene recordar que el IPN se organizó en tres niveles educativos que entrañan una marcada estratificación laboral y social: prevocacional orientado a adiestrar aprendices y obreros calificados, vocacional dirigido a instruir técnicos o supervisores, y superior dedicado a formar ingenieros y directores técnicos.

\section{Los Institutos Tecnológicos}

En 1948 se creó el Instituto Tecnológico de Durango y en 1949 el Instituto Tecnológico de Chihuahua (Hernández, 1996), los primeros de los que más tarde configurarían la Dirección General de Enseñanzas Tecnológicas Industriales y Comerciales (DGETIC), al separarse en 1960 del IPN e integrarse a la Secretaría de Educación Pública (SEP). La condición central de estos Institutos era su carácter regional, representando la apuesta necesaria exigida por el modelo económico de Industrialización por Sustitución de Importaciones (ISI) adoptado con diferentes matices en casi todo el territorio latinoamericano durante el período de pos-guerra, con la intención de propiciar la autosuficiencia industrial y el desarrollo regional del país. En el documento de creación, se explicitó que la DGETIC:

Coadyuva al fomento de la industrialización del país, de acuerdo con las características económicas y sociales de cada región, y su acción educativa está encaminada a la formación de técnicos en sus diversos niveles: medio en sus dos ciclos, y superior, sin descuidar las actividades culturales, cívicas, deportivas y sociales, para lograr la educación integral del alumno (SEP, citado en Hernández, 1996: XII).

Resaltan en la cita anterior dos concepciones pedagógicas encontradas, pero no necesariamente opuestas: la formación técnica y la educación integral, las cuales se anudan, en un tejido muy particular, a la tradición heredada del modelo politécnico francés y su base formativo-curricular asentada en el conocimiento científico; esto genera en el nuevo sistema un interesante crisol de tensiones, entre lo nacional y lo regional, lo científico y lo técnico, la teoría y la práctica, la base científica general y la proliferación y heterogeneidad de los programas de estudio, resultantes en parte por la diversidad de las

\footnotetext{
${ }^{4}$ Las cursivas son mías.
} 
demandas regionales y también por la influencia de la tendencia pragmática norteamericana, que no logró, sin embargo, desplazar la lógica cientificista del modelo europeo. El resultado fue una superposición, que no integración, entre la formación básica teórica (común) y las prácticas profesionales-técnicas que componían las curricula de programas de estudio dedicados a la formación de superespecialistas (Weiss y Bernal, 2013).

Entre 1970 y 1980, la educación superior tecnológica recibió un impulso preferencial por parte del gobierno federal, de tal forma que de siete Institutos Tecnológicos y el IPN, existentes en 1969, para 1990 el Sistema contaba ya con más de 100 planteles, con un crecimiento de $242 \%$ en la matrícula de licenciatura. De 1990 a 2010 los Institutos Tecnológicos crecieron de 100 a 228, lo que implicó un aumento de 218\% (Weiss y Bernal, 2013); de 2010 a 2016 el crecimiento fue de $22 \%$, llegando al total actual de 266 instituciones.

Para entender las condicionantes políticas y económicas relacionadas con este crecimiento, es importante hacer mención del proceso de descentralización iniciado en el Sistema a partir de la década de los 90, el cual consistió en:

La "transferencia" del control administrativo y del financiamiento operativo de las escuelas del gobierno federal a los gobiernos estatales, y de desconcentración de la oferta de las ciudades más importantes a localidades más pequeñas y rurales, sobre todo a través de la creación de instituciones educativas de nuevo tipo, denominadas genéricamente "organismos descentralizados de los gobiernos de los estados con participación federal (ODE)” (Weiss y Bernal, 2013: 161).

Bajo esta figura se crean los Institutos Tecnológicos Superiores (ITS), que actualmente han representado el medio privilegiado de crecimiento del Sistema, de tal forma que de 1990 a la fecha se crearon 108 iтs y sólo 10 гт Federales.

Una condición histórica nodal para el entendimiento de los modelos educativos, motivo del presente trabajo, se puede ubicar precisamente en esa década, específicamente en el Programa para la Modernización Educativa 1989-1994, donde "[...] se propuso una reforma de las carreras y planes de estudio de las licenciaturas e ingenierías de los institutos tecnológicos" (Vargas, citado en Weiss y Bernal, 2013: 161).

Para el Sistema Tecnológico, la reforma se centró en recuperar criterios internacionales en la organización curricular, de ello se derivó la compactación de las carreras (de 55 a 19), modificaciones de asignaturas, actualización de contenidos e integración de residencias profesionales a la estructura curricular; además, los it incursionaron en esa década en la oferta de estudios de posgrado (Ruíz, 2011). 
Estas acciones, sumadas a la constante preocupación manifiesta sobre la heterogeneidad y la dispersión del Sistema Tecnológico, generaron las condiciones de posibilidad para dos elementos nodales de la reestructuración del Sistema: en el terreno académico-pedagógico, la creación en 2004 del Modelo Educativo para el Siglo XXI (en adelante MES XXI) que constituyó la antesala del Modelo Educativo para el Siglo xxI, Formación y Desarrollo de Competencias Profesionales (en adelante MES XXI por competencias; DGEST, 2012). En el terreno político-administrativo, la conformación en 2015 del recNM. En los apartados subsecuentes se exploran las condiciones vinculadas al terreno académico-pedagógico, por ser el aspecto de mayor interés para este ejercicio investigativo.

\section{La necesidad de un modelo educativo}

La década de los 90 puede leerse como un parteaguas para el Sistema Educativo Mexicano; no fue el resultado de una sola condición, por ejemplo el Programa para la Modernización Educativa, iniciado por el gobierno neoliberal de Salinas, como podría pensarse desde una lectura causal, sino de una compleja e intrincada trama de condiciones tanto políticas como económicas, sociales, culturales, científicas, tecnológicas y académicas.

En el presente apartado se plantea, con fines analíticos, un bosquejo de algunos de los hilos que componen esa trama, enfatizando en los aspectos que fueron claves para reconocer la necesidad de un modelo educativo que orientara las acciones académico-institucionales en el entonces Sistema Nacional de Educación Superior Tecnológica (SNEST).

Al respecto, cabe señalar que una condición nodal y que involucra de alguna forma todas las dimensiones mencionadas, fue la emergencia de las sociedades de la información y del conocimiento (UNESCO, 2005) ligadas profundamente a la producción y la proliferación de nuevas tecnologías de la información y la comunicación (TIC), las cuales generaron una dinámica social inédita identificada por el rompimiento de las fronteras (geográficas, institucionales, sociales, culturales, disciplinarias) y la rápida diseminación de la información en tiempo real. Esto dio pie a una reconfiguración de las formas de producción del conocimiento caracterizadas en la sociedad industrial por su carácter disciplinario y la estabilidad que le otorgaban las pretensiones de objetividad y verdad asignada a las ciencias; devenían de la tradición positivista, aunadas a la lentitud de su difusión determinada por los procesos editoriales y de impresión necesarios para la elaboración del entonces medio privilegiado de transmisión del saber, el libro. 
El conocimiento instaurado en significante nodal de las nuevas sociedades ha tenido significaciones múltiples, se ha pensado entre otros sentidos como el instrumento necesario para el desarrollo social; como producto de consumo e intercambio, es decir, como la mercancía privilegiada de una nueva configuración económica cuyo vehículo principal son las TIC, a saber, las economías del conocimiento (Steinmueller, 2002).

Estas significaciones del conocimiento coadyuvaron a que distintos organismos internacionales ${ }^{5}$ centraran su atención en las actividades de producción, conservación y difusión del conocimiento y, por supuesto, en las instituciones encargadas de ello, lo que derivó en diversas "recomendaciones" sobre la necesaria revisión y reestructuración de los sistemas educativos nacionales, con énfasis especial en las Instituciones de Educación Superior (IES). Al respecto Díaz Barriga (2015: 3) señala que:

En la década de los años 90 se establece bajo la lógica de la globalización, de la economía internacional, de la participación de nuestro país en la OCDE, de la influencia del discurso educativo de los organismos internacionales (BM y BID) una serie de acciones que configuran un modelo que desde esos años, todos los gobiernos han aplicado.

Las instituciones de educación superior tecnológica representaron condiciones muy particulares producidas en su trayectoria histórica y por su énfasis en la técnica como aplicación del conocimiento científico, evidente en la separación estructural entre la base científica y la práctica en sus planes y programas de estudio.

Esto produjo una tensión latente que ha dejado huellas en los modelos educativos objeto de nuestro interés, esto es, entre la consideración tradicional de lo tecnológico y lo técnico como resultante de una apropiaciónaplicación de los conocimientos producidos por otros y la cada vez más urgente necesidad de cuestionar este carácter aplicativo que separa la ciencia de la tecnología y asumir la responsabilidad de propiciar - mediante sus procesos formativos - que los profesionales en ciernes se asuman como potenciales productores de conocimiento que, en su estatus de elemento nodal en el desarrollo de la sociedad y en la reconfiguración económica mundial, se ha constituido en el objeto mercancía privilegiado del capitalismo, como anticipó Lyotard (1979).

\footnotetext{
${ }^{5}$ Banco Mundial (BM), Banco Interamericano de Desarrollo (BID), Organización de Comercio y Desarrollo Económico (OCDE), Organización de las Naciones Unidas para la Educación, la Ciencia y la Cultura (UNESCO), entre otros.
} 
Otro elemento nodal en este escenario lo constituyen las nuevas formas de organización construidas a partir del avance de la cibernética y la informática, cuyo paradigma fundante es la world wide web (www); sus aspiraciones del mundo interconectado se extendieron a distintos campos del quehacer humano, creando nuevas condiciones de posibilidad para ser y actuar en el mundo, pero también nuevos retos basados en la complejidad y en la incertidumbre, signos de la época. Esta aspiración se relacionó estrechamente con la globalización y las sociedades del conocimiento.

La www brindó facilidades sin precedentes para el intercambio de información y conocimiento, permitiendo que las redes producidas como el nuevo y privilegiado formato desafiaran los límites tradicionales que habían circunscrito las formas tradicionales de organización social, planteando estructuraciones distintas, no lineales ni jerárquicas, más bien rizomáticas (Deleuze y Guattari, 1976), esto es, sin centro, por lo que cualquier elemento puede asumir esa función.

Estas condiciones han transformado el panorama mundial, de lo que resultan sociedades muy distintas a la sociedad industrial sobre la que está sentado nuestro sistema educativo, esto es, la sociedad para la cual se está educando y que ya no existe más. Esta condición acentúo la preocupación y los intereses de diversos países sobre los sistemas educativos, generando en Europa el proceso de Bolonia que tenía como propósito construir un marco común donde convergieran los sistemas de educación superior de distintos países, lo que permitió el intercambio de estudiantes y egresados. Esto implicó la necesaria adaptación de los estudios universitarios a las demandas sociales y económicas que se estaban gestando; de ahí se derivó el proyecto Tunning (2000), con el objetivo de establecer condiciones para la movilidad, la cooperación y la convalidación de créditos entre los países que suscribieron el acuerdo. La importancia del proyecto Tunning, en el contexto de este análisis, tiene que ver con la noción que estructura la apuesta académica del modelo educativo vigente en el TесNм: competencias profesionales.

Cabe recordar que este término no es nuevo, ya en la década de los 70 se había usado en el contexto educativo, específicamente en Estados Unidos de Norteamérica y Canadá, países en los que las competencias habían sido entendidas desde una connotación reducida, orientada a enfrentar la crisis económica de los 70 a partir del entrenamiento y la capacitación, para que los jóvenes estuvieran en condiciones de responder a las demandas del mercado laboral.

En México esta perspectiva no fue ajena, se introdujo desde principios de los 90 a través del Colegio Nacional de Educación Profesional Técnica (CONALEP) y se fue diseminando por todo el Sistema Educativo, especialmente en los subsistemas relacionados con la educación técnica y tecnológica. 
La noción de competencias profesionales presenta huellas muy marcadas de esta concepción.

Esta situación generó condiciones de posibilidad para la construcción del primer modelo educativo; cabe señalar que antes de éste hubo varios intentos de constituir un modelo que diera sentido a las concepciones y acciones del Sistema, pero ninguno llegó a concretarse en un modelo, hasta 2004.

\section{Continuidades discursivas en los Modelos Educativos del TecNM}

El MES XXI fue el primer intento de organización académico curricular del SNEST que se cristalizó en un documento de observancia nacional. El SNEST hasta entonces se caracterizaba por la multiplicidad de modelos y propuestas curriculares que devenían en la dispersión de programas de estudio y en la ausencia de ejes rectores que permitieran la articulación entre las instituciones, condición que dificultó la construcción de una base conceptual común de sus procesos formativos.

Vale la pena reconocer que el MES XXI (SNEST, 2004) y el MES XXI por competencias (DGEST, 2012) representan el inicio de un camino que aún se está construyendo, de ahí la relevancia de ejercicios analíticos que los tomen por objeto.

En ese sentido, el análisis realizado ha puesto en relieve algunas continuidades discursivas, entendidas como elementos que atraviesan los modelos revisados, imprimiendo huellas tanto en las estructuras como en las propuestas conceptuales y procedimentales. Son consideradas continuidades en tanto representan elementos que han permanecido, sin variaciones significativas, en los tres modelos; algunos han estado presentes desde los principios mismos de la educación técnica y tecnológica en el país, creando una ilusión de universalidad que limita su problematización.

\section{Pensamiento moderno}

Es importe ubicar en la base del MES XXI las ideas propias de la modernidad y sus principios ilustrados, lo que marca una línea de continuidad con la historia de la educación técnica y tecnológica en México. Después de reconocer que las necesidades y los escenarios sociales han cambiado, el texto declara como exigencia " [...] el renacimiento del proyecto de la modernidad, estan- 
cado actualmente entre el desencanto ideológico posmoderno y la deshumanización de las economías del mundo" (SNEST, 2004:9).

Esta declaración evoca la apuesta habermasiana que invita a reconocer el proyecto moderno como inacabado, por lo que es posible reconstruirlo partiendo de una nueva racionalidad que, sin dejar a un lado los ideales y principios de la modernidad, permita rectificar los pasos que llevaron a su fracaso (Santana y Pérez, 1999).

Esta base moderna se asume sin mayores revisiones en los dos modelos educativos publicados posteriormente. El riesgo de esta postura estriba en que no se problematizan las bases del pensamiento moderno, se asumen en su carácter de verdad y se quiere aplicarlos sin más a una sociedad distinta, construida sobre la incertidumbre y el desencanto producido por el fracaso de la razón y las desventuras del progreso que se aspiraba alcanzar mediante la ciencia objetiva y su aplicación técnica, esto es, una ciencia elevada por encima de lo social, lo moral, lo ético, lo político, lo humano mismo.

Si se asume la vigencia del proyecto moderno, sin más, no hay lugar para un cuestionamiento capaz de posibilitar la resignificación de la relación entre ciencia-tecnología y sus implicaciones en la formación de ingenieros. Cabe resaltar que en ninguno de los modelos educativos revisados se explícita un concepto de educación técnica o tecnológica que fundamente la propuesta.

\section{Enfoque sistémico}

Otro aspecto que forma parte de los modelos educativos analizados, es su enfoque sistémico, de ahí que la estructura se conforme por tres dimensiones interactuantes, a saber, la dimensión filosófica, la académica y la organizacional, en las que tienen lugar cinco procesos: académico, vinculación, planeación, administrativo, calidad. Esta estructura sistémica es la misma en los tres modelos analizados.

En el MES XXI (SNEST, 2004) se explícita y reitera constantemente la interacción entre las dimensiones y los procesos de la estructura, sin embargo brinda pocas pautas para entender y sobre todo poner en marcha estas interacciones.

En el MES XXI por competencias (DGEST, 2012) se describen de manera más amplia y se da contenido a los procesos que ya habían sido enunciados en 2004, pero no se propone un cambio sustancial en la manera de entender el proceso educativo, no obstante, se señalan nuevos puntos de intersección que parten de la dimensión organizacional, que orientan las acciones de las otras dos dimensiones. 
El Modelo de Educación Dual para nivel licenciatura del Tecnológico Nacional de México (en adelante MEDTecnM) explicita que se basa en la misma estructura organizadora de los modelos anteriores, por lo cual sintetiza en qué consiste cada una de las dimensiones de esta estructura, sin embargo, en el desarrollo de la propuesta estas dimensiones estructurales son obviadas y en su lugar se desarrolla un conjunto de procedimientos (a manera de manual de operaciones) de cómo llevar a la práctica el modelo basado en la vinculación escuela-empresa.

La mirada sistémica, en tanto plantea una condición relacional e interactuante entre distintas dimensiones, es interesante para entender lo educativo, sin embargo sería importante repensar las dimensiones que estructuran el proceso educativo y, sobre todo, replantear las interacciones entre éstas, partiendo de la especificidad de lo educativo, pues como son planteadas en los tres modelos y de manera más contundente en los dos más recientes, la dimensión organizacional parece dominar los campos conceptuales y de acción de las otras dimensiones.

\section{Perspectiva administrativa}

El enfoque sistémico se acompaña de otras dos perspectivas: las teorías de la calidad y la gestión estratégica; ambas atraviesan las dimensiones y los procesos del modelo. Sobre esto, vale la pena enfatizar que el terreno disciplinario del que proceden es la administración, lo que deja huellas profundas en los modelos educativos, reflejadas en su manera de pensar lo educativo como un proceso más cercano a lo empresarial y marcado por las exigencias actuales de la economía mundial.

Esto se entrevé en el sentido que se le asigna a un significante nodal en los tres modelos: calidad, definida como "forma de ser orientada a la mejora continua de los productos, bienes o servicios, sistemas y procesos del Modelo, con el propósito de crear valor para sus beneficiarios" (SNEST, 2004: 40). Este concepto administrativo de calidad no se problematiza; en los tres modelos se asume constantemente, se reitera sin definición y, aunque se plantea en términos de calidad educativa, no hay una construcción conceptual en ese sentido.

En la dimensión académica se señalan aspectos relacionados con la formación profesional y se enfatiza en las experiencias de aprendizaje y la práctica educativa como formación integral; sin embargo, la impronta de la mirada administrativa, bajo la cual queda soterrada la necesidad de una mirada pedagógica, es evidente en conceptos como capital humano (para referir a 
los actores que forman parte del proceso) y en la constante preocupación por los indicadores y estándares que permitan medir con objetividad la mejora continua de los productos y servicios que el sistema ofrece. Quizá esta preocupación explique la ausencia de problematizaciones o al menos conceptualizaciones de lo educativo y de la educación superior tecnológica, necesarias para fundamentar y orientar un modelo educativo dirigido principalmente a la formación de ingenieros.

Un modelo educativo debe situar como eje de su estructura una lógica educativa-académica y pedagógica que oriente sus concepciones y acciones, por lo que es importante resignificar la dimensión académica del modelo, que en los tres casos parece quedar supeditada a la lógica administrativa. Esta situación se hace por demás evidente en el caso del MEDTecNM que se limita a describir procedimientos (casi desde una lógica fabril) con los que se pretende desarrollar el proceso y alcanzar los objetivos educativos.

\section{El concepto de competencias profesionales}

Desde el modelo de 2004, se anticipaba entre líneas el enfoque por competencias, pero es hasta el modelo de 2012 que se concreta la noción de competencias profesionales, eje de la propuesta educativa y curricular del тесNм a partir de entonces. La noción de competencias profesionales conjunta elementos de la concepción de competencias europea, centrada en la formación universitaria, que considera tres componentes de la competencia (conocimientos, habilidades y actitudes), sin superar la concepción norteamericana, centrada en el entrenamiento laboral, reduciendo la competencia a uno de sus componentes enfocadas las habilidades en el saber hacer.

Esta yuxtaposición de perspectivas en la noción de competencias tiene matices específicos que, tensados por la mirada administrativa, decanta el proceso educativo hacia la capacitación laboral. Justo sobre esa concepción se basa la propuesta del MEDTесNM, centrada más en los intereses empresariales que en el proceso formativo, de ahí que se pretenda constituir en "[...] un modelo flexible formativo altamente influenciado por los empleadores que propicie el aprendizaje de los estudiantes y promueva su incorporación a la vida laboral y a los procesos productivos del sector empresarial" (TecNM, 2015: 1).

Desde esta concepción, aunque no es explícito, la formación del ingeniero se reduce a un entrenamiento técnico, pues más que una filosofía de la educación desde la que se considera como fin educativo la formación de sujetos, lo que opera es una filosofía de mercado donde el estudiante es concebido como un recurso más de la empresa, por ello lo que importa es que esté ha- 
bilitado técnicamente para desarrollar eficaz y eficientemente las funciones de su puesto.

El saber hacer conlleva el saber aplicar un conocimiento (técnico) en el desarrollo de determinado procedimiento o la ejecución de un proyecto. En ese sentido, en la noción de competencias profesionales se conserva la distinción moderna, ya rebasada por las necesidades sociales contemporáneas, entre productores y aplicadores del conocimiento.

\section{Tensiones y rupturas conceptuales}

Aunque hay elementos que representan continuidades entre los tres modelos revisados, es importante reconocer que hay tensiones y rupturas conceptuales que implican significaciones distintas. Este apartado está enfocado en poner sobre relieve estas diferencias que son innegables y constitutivas, a pesar de que pudiera pretenderse una línea de desarrollo, lineal y ascendente, entre ellos.

Estas diferencias están presentes en las distintas dimensiones estructurales de los modelos, sin embargo por los objetivos de la investigación, este trabajo se centra en la dimensión académica, enfatizando en aquellas que conllevan implicaciones curriculares y se encuentran vinculadas con la concepción de educación tecnológica y formación de ingenieros que subyace en el Sistema.

\section{Formación profesional y responsabilidad social}

Una tensión importante en este sentido tiene que ver con las distintas concepciones de la formación profesional y la responsabilidad social que ésta implica. Por un lado el Modelo Educativo para el Siglo XXI explícita su orientación humanista a la hora de definir la formación profesional, lo cual se traduce en el reconocimiento de la persona como ser integral y único, por lo que la apuesta se centra en el aprendizaje y en los proyectos de vida, en ese sentido sostiene que:

Una de las finalidades fundamentales del proceso educativo es la formación integral, de modo que la educación en el SNEST se concibe como un proceso continuo de desarrollo de todas las potencialidades del ser humano, que lo orienta hacia la búsqueda de su plenitud en el aprender a ser, a hacer, a aprender, a emprender y a convivir, lo cual lo convierte en un ciudadano y un profesionista consciente, responsable y solidario (SNEST, 2004: 28) 
A pesar de que en el MES XXI por Competencias (DGEST, 2012) se enuncia una formación profesional integral basada en tres dimensiones (saber, hacer y ser), plantea una mirada reduccionista de las necesidades sociales entendidas más bien en términos de demandas laborales; hacia éstas se orienta principalmente el concepto de competencias profesionales, derivando en un énfasis notorio sobre el saber-hacer. Esto se hace presente desde su definición del plano social:

El plano social, corresponde al contexto resultante de la dinámica mundial que define las relaciones entre los diferentes actores que construyen y participan en la realidad económica, social, cultural y política. Tales condiciones generan necesidades específicas respecto de la demanda laboral, por lo que el SNIT ha desarrollado un enfoque educativo basado en la formación y el desarrollo de competencias profesionales para responder a ello (DGEST, 2012: 33).

La formación profesional, en ese sentido, está direccionada a la capacitación para que el egresado pueda adaptarse y responder eficaz y eficientemente al mercado laboral, que "[...] como efecto directo de esta realidad [...] demanda profesionales para puestos de trabajo cada vez más cambiantes y competitivos" (DGEST, 2012: 35).

En el MEDTecNm esta condición se acentúa, desde el momento que se enuncia como un modelo "altamente influenciado por los empleadores" (TecNM, 2015: 1) se declara el centro de interés y la reducción del concepto de formación profesional al training. La función social del Sistema, desde esta perspectiva, deja de estar enclavada en el desarrollo nacional (económico y social) para concentrarse en "[...] la necesidad de mejorar los procesos de formación del capital humano por la demanda de personal calificado" (тесNм, 2015: 14). El riesgo de esta apuesta es que la formación profesional queda supeditada a los requerimientos e intereses de la empresa, que a menudo se reducen a la capacitación técnica e instrumental.

\section{Concepciones implícitas de la educación tecnológica: ¿Transmisión o generación del conocimiento?}

La apuesta al pensamiento moderno declarada en el MES XXI y de la cual ninguno de los modelos posteriores ha tomado distancia explícita, como se argumentó en el apartado anterior, conlleva una perspectiva curricular 
más cercana al modelo politécnico francés, la cual ha estado presente desde los inicios de la educación superior tecnológica en México. Esto se traduce en estructuras curriculares basadas en una consideración de la tecnología como aplicación del conocimiento científico; de ahí que se sitúe en la base curricular la adquisición del conocimiento disciplinario y en otros espacios, principalmente en la residencia profesional, se procure la aplicación de ese conocimiento.

Esta forma de pensar la estructura curricular, si bien ha permanecido en los tres modelos, se ha enfrentado a tensiones y replanteamientos a partir de las condiciones actuales relacionadas con la economía del conocimiento (Steinmueller, 2002) y las reconsideraciones de la relación entre ciencia y tecnología, pues un modelo formativo de ingenieros pertinente a las necesidades socioeconómicas actuales no puede seguir sosteniéndose en la transmisión-adquisición-aplicación del conocimiento sino más bien apuntar a la generación de conocimiento y la innovación como base de la educación tecnológica.

En ese sentido, el MES XXI por competencias intenta incorporar en sus planteamientos nociones como emprendimiento e innovación, además de integrar "[...] asignaturas de especialidad en módulos interdisciplinarios, que incorporan las tendencias tecnológicas emergentes y atienden las necesidades locales, regionales y nacionales" (DGEST, 2012: 49).

Sin embargo, el tránsito de una mirada dicotómica a una integradora para pensar las relaciones entre ciencia y tecnología en la significación de la educación superior tecnológica, no ha logrado completarse. Esto se hace evidente en la estructura curricular basada en la separación entre conocimiento científico (que el estudiante adquiere) y en la aplicación técnica de éste, y en la concepción de competencias profesionales cuya cualidad académica está dirigida a "[...] que la persona adquiera, valore, integre y aplique de forma estratégica un conjunto de conocimientos, procedimientos y actitudes que den sustento a un desempeño pertinente, eficiente y adaptable ante escenarios laborales heterogéneos y cambiantes" (DGEST, 2012: 36), sin dar cabida a la generación o la innovación como parte medular de la formación profesional.

\section{Determinismo vs flexibilidad curricular}

Las concepciones tradicionales de educación escolar, basadas en la idea de transmisión, conllevaron históricamente estructuras curriculares deterministas, esto es, desarrolladas sobre el supuesto de que pueden contener todo el 
conocimiento necesario y que éste es aprendido de maneras más o menos homogéneas por los estudiantes, de lo que resultan planes y programas de estudio rígidos, a partir de los cuales la función del profesor se reduce a la reproducción y los contenidos son considerados verdades incuestionables, absolutas; esto deja poco margen de acción y reflexión, limitando la formación del pensamiento científico en los estudiantes.

El advenimiento de las sociedades y economías del conocimiento ha dislocado la función de reproducción-transmisión de la escuela, a partir de la inédita velocidad con la que se producen y comunican los conocimientos y de la erosión de las fronteras entre su producción y su consumo. En este sentido, cualquier estructura curricular que pretenda determinar apriorísticamente todos los contenidos escolares está destinada a la obsolescencia.

Desde la pedagogía se empezó a usar el término flexibilidad a partir de la década de los 70 del siglo pasado, ante la preocupación por sistemas educativos, estructuras curriculares y planes de estudio verticales (Escalona, 2008), descontextualizados y ahistóricos, incapaces de responder a las necesidades formativas planteadas por las condiciones (sociales, culturales, políticas, económicas, tecnológicas y científicas) emergentes.

En el campo de la política educativa el concepto de flexibilidad curricular es recuperado en "[...] dos documentos básicos que dan la pauta para construir la educación superior de este siglo y contextualizan la política educativa que hay que seguir" (Escalona, 2008: 151), estos son: la Declaración Mundial sobre la Educación Superior en el Siglo xxI: visión y acción, enunciada en 1998 por la UNESCO, y la Declaración de Bolonia, enunciada en 1999 por un conjunto de ministros europeos. En ambos documentos hay una relación estrecha entre la flexibilidad y la movilidad de los distintos actores educativos en escenarios internacionales.

Es a partir de la consideración de la flexibilidad desde la política educativa internacional que en México el término empiece a ser "usado" en leyes, programas, reformas y modelos educativos. En el Modelo Educativo para el Siglo XXI se argumenta la flexibilidad como base, al señalar que:

El renovado y enriquecido Modelo Educativo de los Institutos Tecnológicos se sustenta, precisamente, en una concepción cada vez más amplia y flexible de la tarea de educar, lo que, seguramente, permitirá elevar la pertinencia y la calidad de la educación que en particular imparten estas instituciones (SNEST, 2004: 8).

En todo el documento se recupera la flexibilidad, en distintos sentidos: relacionada con la tolerancia y la apertura ante distintas formas de pensamiento; 
vinculada a la calidad, la extensión y la equidad (en términos de cobertura) del Sistema; como apuesta a la movilidad estudiantil; relacionada con la innovación, o entendida desde la dimensión administrativa (flexibilidad de procesos). Sin embargo, no hay una toma de posición clara respecto a la flexibilidad curricular.

En el Modelo Educativo para el Siglo xxI, Formación y Desarrollo de Competencias Profesionales (DGEST, 2012) también se plantea la idea de que el modelo propuesto es flexible, el término de flexibilidad es propuesto en primer momento en relación con el propio Sistema y su posicionamiento político en el entorno educativo nacional.

En términos de la flexibilidad curricular, en el documento se hace explícito que "[...] los planes y programas de estudio tienen un diseño flexible y una perspectiva interdisciplinaria que favorecen los aprendizajes amplios y complejos; propician la interacción entre los actores del proceso educativoformativo y dan sustento al trabajo colegiado de los docentes" (DGEST, 2012: 49). Sin embargo, al momento de definir el sentido de esta flexibilidad en el diseño curricular, la propuesta se limita a señalar que "[...] el diseño es flexible porque las asignaturas no tienen entre sí ligas que las condicionen a una secuencia única, ni están compactadas de manera rígida por semestre, a la vez que se ofrecen asignaturas de especialidad y salida lateral" (DGEST, 2012: 49). Esta afirmación denota un reduccionismo en el entendimiento de la flexibilidad que así planteada se confunde con cierto atomismo curricular.

Aunque en el documento se enfatiza y reitera la flexibilidad de los planes de estudio, hay un elemento que genera una contradicción a este planteamiento, a saber, el acento en el necesario apego a estos programas; aunque este apego es matizado con indicaciones sobre la revisión de éstos, no se plantean ni los mecanismos, ni los tiempos, ni los sujetos encargados de esta revisión, lo cual puede devenir en una estaticidad de los contenidos, pues la flexibilidad queda supeditada a las condiciones burocráticas implícitas, sobre todo si se asume esta revisión como un proceso más bien administrativo.

En el MEDTecNm (тесNм, 2015) la flexibilidad es entendida principalmente en relación a las estrategias de vinculación entre los Institutos Tecnológicos y las empresas, organizaciones o dependencias gubernamentales, consideradas como las demandantes de fuerza laboral calificada. De ahí que se plantee que la flexibilidad del modelo radica en ser "altamente influenciado por los empleadores" (тесNм, 2015: 19). La formación flexible, desde esta perspectiva, consiste en que esté planificada para que pueda responder "a las necesidades del sector productivo" (тесNм, 2015: 25). 


\section{Lo multi, inter y transdisciplinario}

El entendimiento de las relaciones disciplinarias es un aspecto importante de visibilizar si se pretende un análisis de la formación de ingenieros en el TeCNM, a partir de la revisión de los modelos educativos que han orientado su quehacer formativo, esto por dos cuestiones: 1) la ingeniería como profesión, históricamente se ha caracterizado por ser el espacio de encuentro de distintas miradas disciplinarias, tecnológicas, técnicas, económicas y sociales; 2) la producción del conocimiento en las sociedades actuales se caracteriza por el creciente desbordamiento de las fronteras y los límites disciplinarios.

En el MES XXI (SNEST, 2004) esta relación entre disciplinas no se aborda explícitamente, la única mención que se hace tiene que ver con la complejidad de los problemas y escenarios actuales, a lo que ningún campo disciplinario puede responder por sí solo; en ese sentido, hay un entendimiento implícito de las relaciones disciplinarias relacionadas con "la creatividad, la iniciativa y el desarrollo de competencias para plantear y resolver problemas" (SNEST, 2004: 26). Esta mirada resulta muy limitada pues deja de fuera una discusión epistemológica básica que tendría que ser contemplada para construir un posicionamiento válido sobre la formación de ingenieros.

En el MES XXI por competencias (DGEST, 2012) aunque la cuestión de las relaciones disciplinarias es mencionada en diversas ocasiones (19 menciones para ser precisos), esto es poco problematizado y diferenciado, por un lado porque no hay clarificación entre lo multidisciplinario y lo interdisciplinario, conceptos planteados como sinónimos a lo largo del documento y, por otro, porque en su definición de contenidos sigue priorizándose una lógica disciplinaria, la cual es explícita cuando se sostiene que los contenidos conceptuales (el saber):

Son de carácter disciplinario y permiten describir, explicar, predecir y comprender eventos, fenómenos, relaciones y procesos de la formación profesional, del ejercicio de la profesión, así como de la evolución y la trascendencia de ésta. Los contenidos conceptuales son el fundamento de los contenidos procedimentales (DGEST 2012: 41)

Esto hace evidente por lo menos tres contradicciones: 1) la consideración de la tecnología como aplicación de los conocimientos científicos sostenida sobre la dicotomía teoría-práctica, que contradice las pretensiones de formar profesionales que generen conocimiento e innovaciones; 2) la preminencia de la transmisión de saberes disciplinarios como base de la formación, a pesar de 
que se menciona que la perspectiva de aprendizaje en que se basa el modelo es constructiva; 3) el énfasis disciplinario en la concepción del conocimiento, y aunque se reitera la multi e interdisciplina, éstas quedan referidas, más que a la construcción compleja del conocimiento, a condiciones (políticas y administrativas) impuestas a los grupos de trabajo.

En el MEDTесNм (тесNм, 2015), la discusión sobre las relaciones disciplinarias nuevamente es una cuestión ausente del documento; se hacen algunas menciones, sin clarificación ni diferenciación de la interdisciplinariedad y la multidisciplinariedad, referidas a una condición que debe ser abordada por los grupos de trabajo.

Es importante hacer notar que en ninguno de los documentos analizados se hace mención de la transdiciplinariedad, significante que refiere a las profundas articulaciones disciplinarias que se están produciendo a partir del desbordamiento de los límites que circunscribían las fronteras de la producción del conocimiento, para construir abordajes complejos y holísticos de la realidad-realidades.

\section{Conclusiones}

A lo largo de estas páginas se ha sostenido que en ninguno de los tres modelos educativos del TecNm hay conceptualización o, por lo menos, alguna definición explícita respecto a dos nociones necesarias de ser consideradas en un Modelo que pretende orientar un Sistema que tiene como principal oferta educativa las ingenierías: Formación de ingenieros y Educación Superior Tecnológica.

La ausencia de ejercicios reflexivos y problematizadores dirigidos a la conceptualización de la formación de ingenieros y la educación superior tecnológica, revela no una falta de significación de estas nociones sino el supuesto de universalidad de las mismas, esto es, se asumen desde la consideración de un significado absoluto, pleno de sentido, dado, negando el carácter político e histórico que las construye; esto genera una concepción anacrónica que ha limitado las posibilidades de responder a las condiciones de las sociedades contemporáneas, que no son únicamente las vinculadas con el mercado laboral.

El significado absolutista desde el que es entendida la educación superior tecnológica y la formación de ingenieros se basa en separaciones dicotómicas, propias del pensamiento moderno, entre ciencia y tecnología, teoría y práctica, generación y aplicación de conocimientos. De esto resulta el entendimiento de la tecnología como la mera aplicación de los conocimientos 
científicos, aplicación que parte de una técnica irreflexiva y servil; esta perspectiva ha sido fuertemente debatida en las comunidades científicas desde mediados del siglo pasado, sobre todo a partir de que la fe ciega en la ciencia positiva como base del progreso demostró sus fallas (léase segunda guerra mundial, riesgos nucleares, catástrofes ambientales). Lo anterior derivó en miradas cuestionadoras de esas dicotomías, desde donde se replantearon las relaciones entre ciencia y tecnología, mediadas por condiciones sociales, políticas y éticas.

Es importante hacer evidentes estas significaciones soterradas porque sobre ellas se está sosteniendo el proceso formativo desarrollado en los Institutos Tecnológicos; esto incluye las concepciones de los procesos y los actores educativos, la definición de las estructuras curriculares, el diseño de planes y programas de estudio y las estrategias pedagógicas.

Construir una conceptualización de la educación superior tecnológica y la formación de ingenieros que considere las dinámicas y complejas relaciones existentes entre la ciencia y la tecnología, la producción y el consumo de conocimientos y las exigencias sociales contemporáneas, brinda posibilidades de fundamentación para la generación y la puesta en marcha de un modelo educativo que coadyuve en el desarrollo de procesos formativos pertinentes a los contextos actuales y que permitan al TесNM alcanzar y consolidar su trascendente misión.

\section{Referencias}

Deleuze, Gilles y Guattari, Félix (1972). Rizoma (Introducción a Mil Mesetas). Valencia (1997), ed. Pre-textos. 64 págs.

DGEST (2012). Modelo Educativo para el Siglo XXI, Formación y Desarrollo de Competencias Profesionales. Ciudad de México, Dirección General de Educación Superior Tecnológica. 102 págs.

Díaz Barriga, Ángel (2015). Redefinir el modelo educativo ¿Tarea de dos años? En Distancia por Tiempos, Nexos-Blog de educación. Revisado en julio de 2016 en: http:/ / educacion.nexos.com.mx/?p=42

Escalona, Lina (2008). Flexibilidad Curricular, elemento clave para mejorar la educación bibliotecológica. En Investigación bibliotecológica, vol. 22, número 44. Ciudad de México, unam. Pp. 143-160.

Flores, Eduardo (2004). Tiempo y Sociedad, en el Real Seminario de Minería, 17921821. En revista brasileira de história da educação nº 8 jul./dez. Maringá, SBHE. Pp. 225-242.

Hernández, Emiliano (1996). Los Institutos Tecnológicos Regionales- Educación Técnica Superior para la Provincia Mexicana. Durango, Instituto Tecnológico de Durango-Patronato Promotor del Desarrollo del ITD. 646 págs. 
IPN (s/f). Página Electrónica del Instituto Politécnico Nacional. Consultado en línea el 15 de enero de 2016 en http://www.ipn.mx/Acerca-del-IPN/Paginas/Historia.aspx

Lyotard, Jean Francoise (1979). La Condición Posmoderna, informe sobre el saber. Madrid, Ediciones Cátedra ( $2^{\mathrm{a}}$ ed, 1987). 68 págs.

Ruíz-Larraguivel, Estela (2011). La Educación Superior Tecnológica en México: Historia, Situación Actual y Perspectivas. En Revista Iberoamericana de Educación Superior, Vol. 2 núm. 3. México, iIsSue-unam/universia. Consultado el 25 de febrero de 2016 en http:/ / ries.universia.net/index.php/ries/article/view/79

Sandín, María Paz (2003). Investigación Cualitativa en Educación. Fundamentos y tradiciones. España, McGraw Hill. Págs. 259.

Santana, Juan Manuel y Pérez, Antonia (1999). Habermas y Foucault, Modernidad, Posmodernidad y Teoría de la Historia. En Vegueta, No. 4. Gran Canaria, unLP. Pp. 103-116. Consultado en agosto de 2016 en http:/ / acceda.ulpgc.es/bitstre am/10553/2373/1/0234500_00004_0006.pdf

SEDena (s/f). Página Electrónica de la Secretaría de Defensa Nacional. Consultada en noviembre de 2016 en http:/ / www.gob.mx/sedena/acciones-y-programas/ sistema-educativo-militar

SNEST (2004). Modelo Educativo para el Siglo Xxı. Ciudad de México, Sistema Nacional de Educación Superior Tecnológica. Págs. 48.

Steinmueller, W. Edgar (2002). Las economías basadas en el conocimiento y las tecnologías de la información y la comunicación. Revista Internacional de Ciencias Sociales, núm. 171, oEI. Pp. 1-17. Consultado en diciembre de 2016 en: http:/ / www.oei.es/historico/salactsi/rics171.htm

тесnм (s/f). Página Electrónica del Tecnológico Nacional de México, consultada en marzo de 2016 en: http://www.tecnm.mx/informacion/sistema-nacionalde-educacion-superior-tecnologica

TecNM (2015). Modelo de Educación Dual para el Nivel Licenciatura del Tecnológico Nacional de México. Ciudad de México, Tecnológico Nacional de México. unEsCo (2005). Hacia las sociedades del Conocimiento. Consultado en enero de 2016 en: http:/ / unesdoc.unesco.org/images/0014/001419/141908s.pdf

Weiss, Eduardo y Bernal, Enrique (2013). Un Diálogo con la Historia de la Educación Técnica en México. En Perfiles Educativos, vol. xxxv, núm. 139, 2013. Ciudad de México, issue-unam. Pp. 151-170 